\title{
An Australian Real-World Study of Treatment Persistence of Ustekinumab in Crohn's Disease
}

\author{
Tzu Hsiang Chien' \\ Andrea Puig (D) \\ Thang Khuong ${ }^{2}$ \\ Mahsa $\mathrm{H}$ Kouhkamari ${ }^{2}$ \\ Samuel Che ${ }^{2}$ \\ Tom Hsun-Wei Huang (D) \\ 'Janssen-Cilag Pty Ltd, Sydney, NSW, \\ Australia; ${ }^{2}$ Prospection Pty Ltd, Sydney, \\ NSW, Australia
}

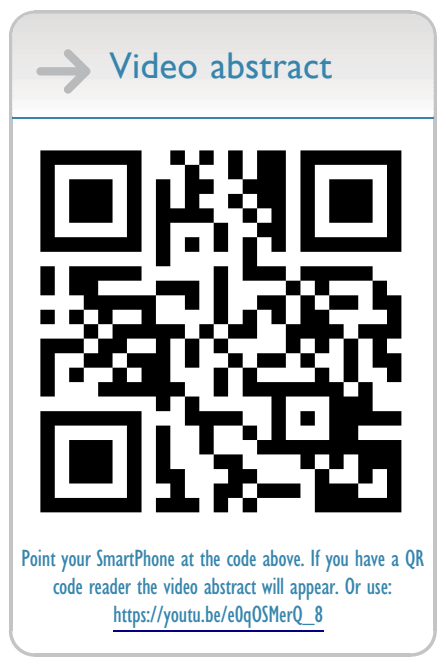

Correspondence: Tzu Hsiang Chien Janssen Australia and New Zealand (Janssen-Cilag Pty Ltd), 66 Waterloo Road, Macquarie Park, NSW, 2113 , Australia

Tel +6I (0)400 919282

Email tchienI@ITS.JNJ.com
Introduction: Real-world treatment persistence to ustekinumab for Crohn's disease (CD) was studied using Australian Pharmaceutical Benefits Scheme (PBS) data. Demographic and treatment pattern characteristics were also investigated.

Methods: Our retrospective cohort analysis included PBS 10\% sample data for ustekinumab from September 2017 to March 2020, and for other biologics from October 2007 to capture earlier line(s) of therapy. Included patients received ustekinumab for CD prescribed by a gastroenterologist. Treatment persistence overall and by prior biologic experience, monoor combination therapy, sex and age were estimated using Kaplan-Meier methods. A Cox proportional hazards regression analysis was performed to assess the effect of age, sex and line of therapy on persistence.

Results: Data were available for 301 patients. Of these, $58.8 \%$ were female and $76.7 \%$ were aged 26-65 years. Median follow-up from first ustekinumab dispense was 16 months. Median persistence to ustekinumab had not been reached. Twelve-month persistence to ustekinumab was $82.6 \%$ (95\% CI 78.1-87.5\%). Patients receiving ustekinumab as their first biologic therapy had 12-month persistence of $88.0 \%(80.8-95.9 \%)$ compared to $80.6 \%(75.0-86.6 \%)$ for patients who had previously received other biologic therapies $(\mathrm{p}=0.059)$. The adjusted analysis showed a trend to longer persistence for patients receiving ustekinumab as their first biologic therapy compared to biologic experienced patients (HR 1.86 (95\% CI 0.95-3.63), $\mathrm{p}=0.070)$. Males had higher persistence to ustekinumab than females (HR $0.36(0.20-0.66), \mathrm{p}<0.001)$. Receiving ustekinumab as a monotherapy or in combination with azathioprine, mercaptopurine, 5ASAs, methotrexate, or corticosteroids had no effect on persistence $(\mathrm{p}=0.22)$.

Conclusion: In an Australian real-world setting, persistence to ustekinumab was demonstrated to be over $80 \%$ at 12 months. Use as monotherapy or in combination with other therapy for CD did not affect persistence. Differences in treatment persistence by gender and previous biologic use warrant further investigation as further long-term data becomes available.

Keywords: persistence, durability, real-world data, Crohn's disease, ustekinumab

\section{Introduction}

Inflammatory bowel disease (IBD) is a general diagnostic term that describes conditions with idiopathic, chronic, relapsing and remitting inflammation of the gastrointestinal tract. Crohn's disease (CD) and ulcerative colitis (UC) are the two most common types of IBD. CD causes abdominal pain, weight loss, fever, diarrhea, rectal bleeding and fatigue and has an unpredictable relapsing and remitting course. Patients are commonly diagnosed in adolescence and early adulthood, and about 1 in 250 people aged 5-40 years in Australia has CD. The prevalence and severity of IBD in Australia is increasing and is one of the highest in the world. ${ }^{1,2}$ 
$\mathrm{CD}$ is currently incurable and the aim of therapy is to induce and maintain remission, ${ }^{3}$ treat acute disease, improve and maintain quality of life, correct nutritional deficiencies and prevent complications, hospitalizations and surgery. ${ }^{4}$ In Australia, patients with moderate to severe CD are generally initially treated with systemic corticosteroids, followed by maintenance with immunomodulators such as azathioprine, mercaptopurine or methotrexate. Patients who are refractory to immunomodulator therapy despite dose optimization are considered for biologic therapy (anti-tumor necrosis factor (anti-TNF) therapy, or vedolizumab or ustekinumab). Biologic therapies can also be introduced early where patients have an aggressive disease course or poor prognostic factors and can be used in combination with corticosteroids and immunomodulators to increase the efficacy of treatment. ${ }^{3}$ In Australia, this does not generally occur due to the subsidization criteria of biologic therapies for $\mathrm{CD}$.

Ustekinumab, an anti-IL12 and anti-IL23 monoclonal antibody, is the most recently available biologic therapy. It is indicated for the treatment of adult patients with moderate to severe active $C D$ who have had an inadequate response, have lost response, or were intolerant to either conventional therapy or a TNF $\alpha$ antagonist or have medical contraindications to such therapies. Ustekinumab is initiated with a single IV dose followed by a $90 \mathrm{mg}$ subcutaneous dose 8 weeks later and regular maintenance dosing every 8 weeks. ${ }^{5}$ Consensus guidelines recommend ustekinumab as a treatment option for the induction or maintenance of remission, both in patients for whom antiTNF treatment fails or in anti-TNF-naïve patients. ${ }^{3,6}$ These guidelines recognize the growing real-world experience of ustekinumab, and support its clinical effectiveness. ${ }^{3,7,8}$

Ustekinumab has been subsidized by the Australian Government through the Pharmaceutical Benefits Scheme (PBS) for CD since September 2017..$^{5}$ The PBS is an Australian Government scheme that provides subsidized prescription medicines to residents. An application for PBS reimbursement of ustekinumab is completed by the patient's physician to initiate treatment and ongoing assessment of continued suitability occurs every 24 weeks. Ustekinumab is only reimbursed in Australia for use in CD.

In Australia, real-world data on prescribing of medications can be obtained through a $10 \%$ sample of PBS pharmaceutical claims data, made available for research by the Australian Government via data custodians. It is a longitudinal systematic random sample of $10 \%$ of the Medicare-eligible Australian population and all of their reimbursed dispensations. ${ }^{9}$ The $10 \%$ PBS dataset captures community-based prescriptions of eligible prescription medicines prescribed under the scheme. ${ }^{10}$

There are a very limited number of Australian real-world studies of ustekinumab. A study comparing the persistence of biologics for UC and CD using PBS 10\% data from 2005 to 2019 found for patients with $\mathrm{CD}$, persistence to a biologic agent was higher in patients administered ustekinumab than vedolizumab, adalimumab or infliximab. ${ }^{11}$

In this study, we investigated persistence to ustekinumab therapy for CD using PBS $10 \%$ sample data from September 2017 to March 2020. Persistence was defined as the duration of time from initiation to discontinuation or switch of therapy and provides an indirect measure to examine long-term clinical outcomes of medications. Persistence to ustekinumab when used as the first biologic therapy for $\mathrm{CD}$ was compared to persistence when it was used in later lines of therapy and persistence to ustekinumab as a monotherapy was compared to ustekinumab used in combination with other therapies for CD. Demographic and treatment pattern characteristics of patients receiving ustekinumab for CD in Australia were described.

\section{Methods}

This study was a retrospective cohort analysis using the Australia Department of Human Services PBS 10\% sample data. Data from October 2007 to March 2020 were extracted and analyzed by Prospection Pty Ltd in their role as the data custodian. This study and publication of subsequent results were approved by the Australian Government Department of Human Services External Request Evaluation Committee (Approval number RMS 1618, 15th April 2021) and complied with relevant data protection and privacy regulations.

\section{Patient Selection}

Data were included for the prescription of ustekinumab from the period September 2017 to March 2020. This date was selected as it corresponded to the date when ustekinumab was first listed on the PBS in Australia for severe CD. Criteria were applied to ensure the included patients had a diagnosis of $\mathrm{CD}$ and were unlikely to have a diagnosis of $\mathrm{UC}$ or other condition treated with ustekinumab.

Patients were included if they had seen a gastroenterologist. In circumstances where ustekinumab was coded to $\mathrm{CD}$, but prescribed by a rheumatologist or dermatologist, it was assumed that this was miscoding of treatments at the pharmacy level and the patient was 
excluded. This is because ustekinumab is also listed on the PBS for the treatment of psoriasis and psoriatic arthritis. Where other therapies were prescribed with an indication of $\mathrm{UC}$, a majority rule was applied to manage potential miscoding between diagnoses of UC and CD. Ustekinumab is not listed on the PBS for UC, so patients prescribed ustekinumab as their first biologic therapy or alone, were unaffected by this.

The most frequent indication over the time period was assumed to be the actual indication. For example, if a patient had 10 scripts allocated to $\mathrm{CD}$ and five scripts allocated to UC, regardless of biologic, all 15 scripts would be allocated to CD.

Patients who received ustekinumab as their first biologic therapy for CD were considered "biologic-naïve" patients and those who had received other biologic therapy prior to receiving ustekinumab for $\mathrm{CD}$ were considered "biologic experienced" patients.

\section{Data}

Data extracted from the dataset included year of birth, sex, state where ustekinumab prescription was filled, PBS item code, concomitant medications and drug dispensing date. The variables used in the analysis included age at initiation (dispensing year minus year of birth), indication (PBS items $11164 \mathrm{~N}, 11182 \mathrm{M}, 11178 \mathrm{H}$ ), line of therapy (calculated on the order of therapy) and previous number of biologic therapies for $\mathrm{CD}$ (adalimumab, infliximab, ustekinumab and vedolizumab).

\section{Statistical Methods}

Use of biologic therapies prior to ustekinumab was determined via analysis of data in the PBS dataset from October 2007 to March 2020. Each time a patient purchased a different biologic therapy for $\mathrm{CD}$, they were considered to have progressed to a later line of therapy. Patients were considered to be biologic naïve if they had no prior biologic therapy for $\mathrm{CD}$ and biologic experienced once they had commenced at least one alternative biologic. The patients on treatment at each line are mutually exclusive.

Patients were summarized by whether they received ustekinumab as a monotherapy or as a combination therapy. Comedication was defined as a purchase of ustekinumab together with one agent of interest within a 6-month period. Concomitant medications of interest included azathioprine, balsalazide, cortisone, dexamethasone, hydrocortisone, mercaptopurine, mesalazine, methotrexate, olsalazine, prednisolone, prednisone sodium phosphate and prednisone. Patients were counted in multiple co-medication groups if more than one combination of medication was taken, that is, combinations are not mutually exclusive.

Treatment persistence was defined as the time, in consecutive days from the first date of prescription of ustekinumab until the last date of prescription prior to a gap of 6 months plus duration of last dispensing (2 months). Patients were assumed to be on treatment until there was a gap of more than 8 months without a script. This reflects a dosing regimen of seven doses of ustekinumab per year at eight weekly intervals consistent with the Australian prescribing information and reimbursement criteria. It includes an induction dose, a second dose at 8 weeks, and assessment of response at week 14-16. It is expected that symptom control should be in evidence by the third or fourth dose (approximately 6 months) and if not, the treating physician will assess the patient for alternatives and a new treatment initiated within the following 2 months. International studies have used similar prescriptive criteria to determine continuation of therapy. ${ }^{12}$

Kaplan-Meier methods were used to estimate treatment persistence. Log rank tests were used to make pairwise comparisons of persistence curves.

A Cox proportional hazards regression analysis was performed to assess the effect of age, gender and line of therapy on persistence. Sensitivity analyses by biologic experience and by combination, or monotherapy at ustekinumab initiation, were included.

All analyses were conducted using Prospection's PharmDash software, except the Cox proportional hazard model was performed using $\mathrm{R}$ version 4.0.2 (https://www. R-project.org/).

\section{Results}

Data in the $10 \%$ PBS sample were available for 301 patients prescribed ustekinumab at least once by a gastroenterologist between September 2017 and March 2020. Median follow-up from time from first dispense of ustekinumab was 16 months. Almost $60 \%$ of the patients were female, most patients were aged between 26 and 65 years and the proportion of patients receiving ustekinumab by state approximated the actual proportion of the population in each state (Table 1).

\section{Treatment Persistence}

Treatment persistence data for 301 patients taking ustekinumab were available from September 2017 to March 2020. The overall 12-month persistence to ustekinumab was $82.6 \%$ (95\% CI $78.1 \%$ to $87.5 \%)$. The 12 - 
Table I Characteristics of Patients with CD Receiving Ustekinumab

\begin{tabular}{|c|c|c|c|}
\hline Characteristic & & n (\%) & \\
\hline \multirow{3}{*}{ Sex } & & & \\
\hline & Male & $124(4 \mid .2)$ & \\
\hline & Female & 177 (58.8) & \\
\hline \multirow[t]{7}{*}{ Age category } & & & \\
\hline & $66+$ & $36(12.0)$ & \\
\hline & $56-65$ & $52(17.3)$ & \\
\hline & $4 \mid-55$ & $82(27.2)$ & \\
\hline & $26-40$ & $97(32.2)$ & \\
\hline & $19-25$ & $33(11.0)$ & \\
\hline & $0-18$ & $\leq 3$ & \\
\hline \multirow[t]{9}{*}{ State* } & & & $\begin{array}{l}\% \text { of Australian population } \\
\text { in each state }\end{array}$ \\
\hline & NSW & $118(39.2)$ & 31.9 \\
\hline & VIC & $79(26.3)$ & 26.0 \\
\hline & QLD & $48(16.0)$ & 20.1 \\
\hline & SA & $23(7.6)$ & 6.9 \\
\hline & WA & $20(6.6)$ & 10.3 \\
\hline & TAS & $5(1.7)$ & 2.1 \\
\hline & ACT & $7(2.3)$ & 1.7 \\
\hline & NT & $\leq 3^{\dagger}$ & 1.0 \\
\hline \multirow[t]{3}{*}{$\begin{array}{l}\text { Prior use of } \\
\text { biologic therapy }\end{array}$} & & & \\
\hline & Biologic-naive & 91 (30.2) & \\
\hline & $\begin{array}{l}\text { Biologic } \\
\text { experienced }\end{array}$ & $210(69.8)$ & \\
\hline
\end{tabular}

Notes: *The numbers in each state are not mutually exclusive as patients may have had ustekinumab dispensed in more than one state; ${ }^{\#}$ Calculated using Australian Bureau of Statistics data for 30 September 2019; ${ }^{\dagger}$ Exact numbers not reported by PBS for categories with three or fewer patients.

month persistence for biologic-naïve patients was $88.0 \%$ $(80.8 \%$ to $95.9 \%)$ and for biologic experienced patients was $80.6 \%(75.0 \%$ to $86.6 \%), \mathrm{p}=0.059$ (Table 2$)$.

The median persistence to ustekinumab overall (Figure 1) and for biologic-naïve and experienced patients (Figure 2) had not been reached. Patients who were biologic naïve prior to receiving ustekinumab had numerically higher persistence to ustekinumab therapy than biologic experienced patients; however, there was no statistically significant difference between the groups. Males had greater treatment persistence compared to females; however, there were no significant differences by age or combination therapy at initiation of ustekinumab.

\section{Cox Proportional Hazards Regression Analysis}

In the adjusted analysis, males were less likely to discontinue than females (HR 0.36 [95\% CI $0.20-0.66] \mathrm{p}<0.001$ ) (Figure 3). Age and prior use of biologic therapy or not, were not significant in the model.

\section{Co-Medication}

More than half of patients (54.7\%) were dispensed ustekinumab as monotherapy (Figure 4). Biologic experienced patients were more likely to receive ustekinumab as a monotherapy (Figure 4). Receiving ustekinumab as a monotherapy or in combination with azathioprine, mercaptopurine, 5 ASAs, methotrexate, or corticosteroids had no effect on persistence $(\mathrm{p}=0.22)$. The 12 -month persistence for patients receiving ustekinumab as a monotherapy was $81.3 \%$ (95\% CI $75.5 \%$ to $87.6 \%$ ) and for combination therapy was $84.9 \%$ (95\% CI $77.8 \%$ to $92.6 \%)$.

\section{Discussion}

The 12-month persistence to ustekinumab was $82.6 \%$ (95\% CI $78.1 \%$ to $87.5 \%$ ). Twelve-month persistence to ustekinumab was greater for patients who were biologic naïve prior to receiving ustekinumab (88.4\%) compared to patients who had received previous biologic therapies (80.4\%). Median time to discontinuation had not been reached; however, the 12-month persistence data showed a trend to greater persistence in patients receiving ustekinumab as their first biologic therapy compared to those who had received previous biologic therapies $(p=0.059)$; however, the log rank test was not significant. The adjusted analysis considering age, gender and prior use of biologics also showed a trend to greater persistence for biologic-naïve patients compared to biologic experienced patients (HR 1.86, 95\% CI 0.95 to $3.63, \mathrm{p}=0.070$ ).

Interestingly, even though across all age groups, the comparison between patients prescribed with ustekinumab as a monotherapy versus combination therapy yielded no statistical difference on persistence (Table 2), males demonstrated higher persistence to therapy than females.

Analyses of persistence to ustekinumab by line of therapy (first line compared to second line and third or later line) were conducted as part of this study; however, they have not been reported separately as the results showed similar trends to those presented for biologicnaïve patients (first line) compared to biologic experienced patients (second or later lines). This may be related to the lower numbers of patients receiving later lines of therapy.

In Australia, there is limited real-world evidence to guide local treatment practice ${ }^{8,11}$ and the $10 \%$ PBS dataset provides valuable insights into the treatment patterns for patients with CD in Australia. A previous analysis of PBS data reported a median ustekinumab persistence rate of approximately $75 \%$ after a maximum follow-up of 2 
Table 2 12-Month Persistence of Ustekinumab by Prior Use of Biologic Therapy, Sex, Age and Combination or Monotherapy

\begin{tabular}{|c|c|c|c|c|c|}
\hline & \multicolumn{5}{|c|}{ I 2-Month Persistence } \\
\hline & & $\mathbf{n}$ & $\%$ & $95 \% \mathrm{Cl}$ & p-value \\
\hline \multirow[t]{3}{*}{ Prior use of biologic therapy } & Overall & 157 & 82.6 & $78.1-87.5$ & \\
\hline & Biologic-naïve & 44 & 88.0 & $80.8-95.9$ & \\
\hline & Biologic experienced & 113 & 80.6 & $75.0-86.6$ & 0.059 \\
\hline \multirow[t]{2}{*}{ Sex } & Male & 71 & 89.8 & $84.2-95.8$ & \\
\hline & Female & 86 & 77.3 & $70.7-84.5$ & $<0.001$ \\
\hline \multirow[t]{5}{*}{ Age, years } & $15-34$ & 55 & 82.8 & $75.2-91.2$ & \\
\hline & $35-44$ & 27 & 76.8 & $64.9-90.9$ & $>0.5$ \\
\hline & $45-54$ & 30 & 90.6 & $82.0-100$ & $>0.5$ \\
\hline & $55-65$ & 25 & 80.1 & $69.7-92.2$ & $>0.5$ \\
\hline & $66+$ & 20 & 82.7 & $7 I . I-96.4$ & $>0.5$ \\
\hline Combination therapy & & 60 & 84.9 & $77.8-92.6$ & \\
\hline Monotherapy & & 55 & 81.3 & $75.5-87.6$ & $>0.05$ \\
\hline
\end{tabular}

Abbreviation: $\mathrm{Cl}$, confidence interval.

years in patients with $\mathrm{CD} .{ }^{11}$ Overall in this study of biologic agents in $\mathrm{CD}$ and $\mathrm{UC}$, longer persistence was observed in males (HR $0.75,95 \%$ CI 0.59 to 0.94 ), and with immunomodulator co-therapy (HR $0.7795 \%$ CI 0.61 to $0.97, \mathrm{p}=0.03){ }^{11}$
In our study, we found a numerically higher persistence in those prescribed ustekinumab as the first biologic therapy compared with those who were biologic experienced. Higher persistence in the first line of therapy has been reported for other biologic agents, ${ }^{13}$ and in other settings,

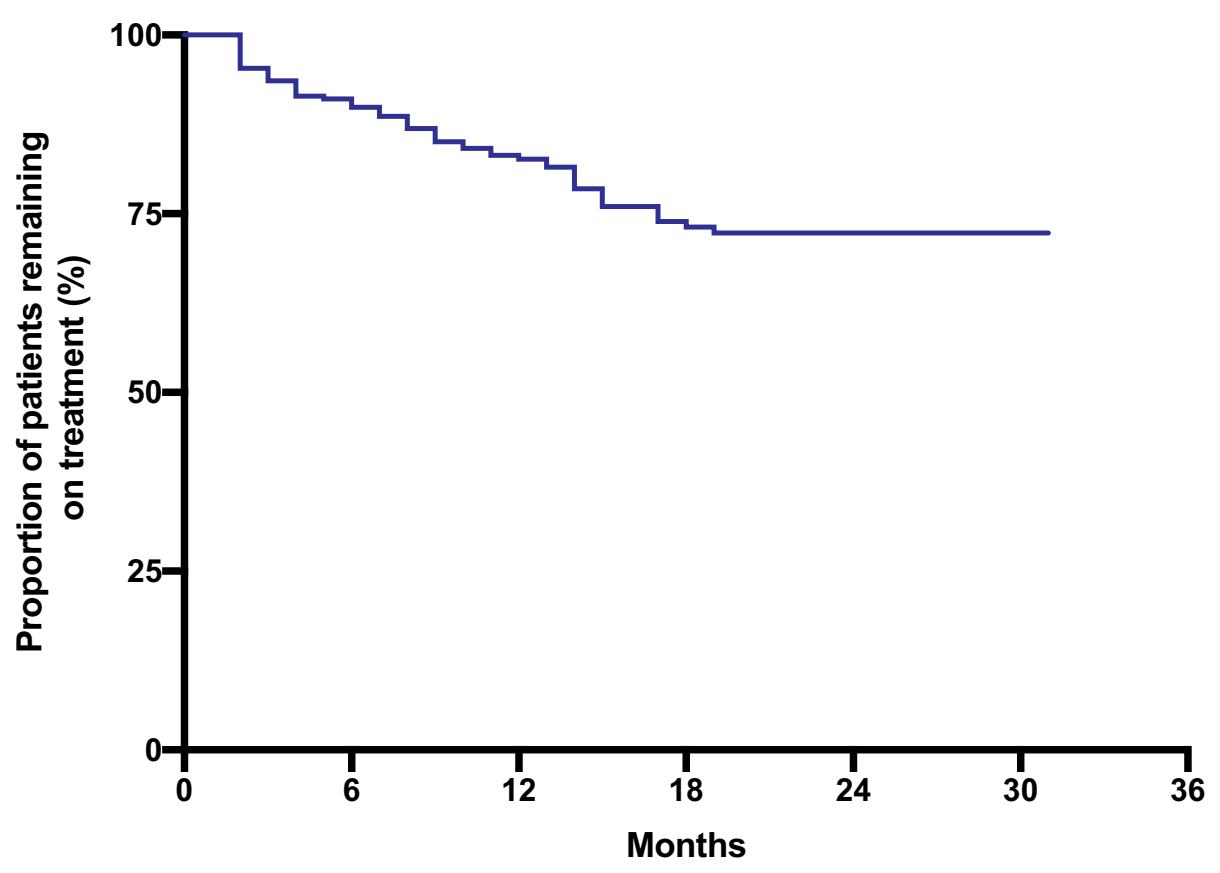

$\begin{array}{lcccccc}\text { Month } & 0 & 6 & 12 & 18 & 24 & 30 \\ \text { At risk } & 301 & 231 & 157 & 95 & 58 & 22\end{array}$

Figure I Kaplan-Meier estimate of overall persistence to ustekinumab. 


\section{Biologic naive}

- Biologic experienced

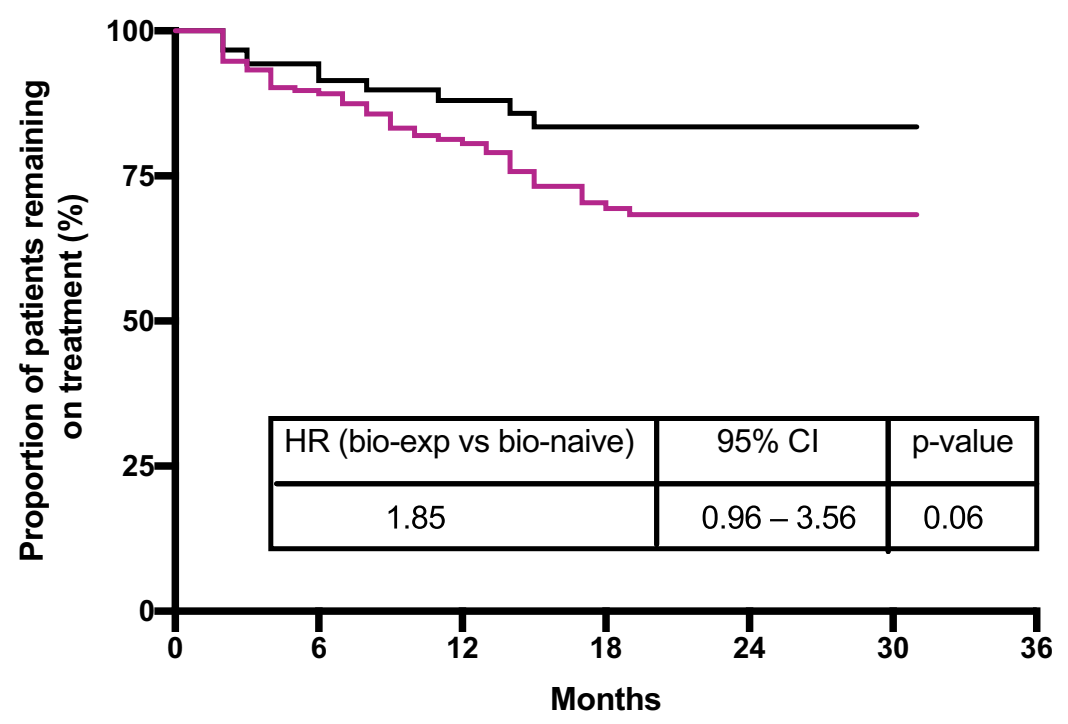

$\begin{array}{lcccccc}\text { Month } & 0 & 6 & 12 & 18 & 24 & 30 \\ & & & & & & \\ \text { At risk } & 91 & 66 & 44 & 25 & 12 & 4 \\ \begin{array}{l}\text { Biologic naïve } \\ \text { Biologic experienced }\end{array} & 210 & 165 & 113 & 70 & 46 & 18\end{array}$

Figure 2 Kaplan-Meier estimate of persistence to ustekinumab by prior use of biologic therapy.

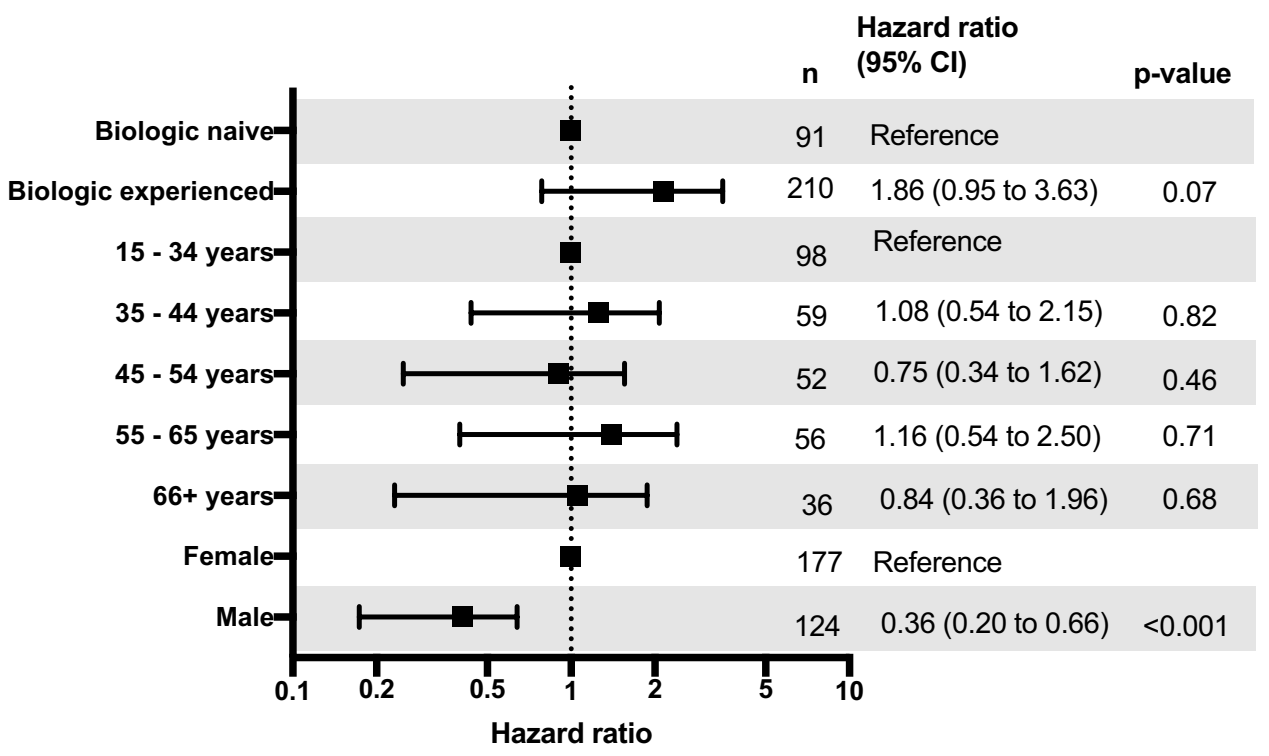

Figure 3 Cox proportional hazards model including age, gender and prior use of biologic therapy.

such as rheumatoid arthritis. The exact reason for this is unknown, though it may be due to secondary non-response to the drug's mechanism of action, inadequate drug levels or antibody formation. ${ }^{14,15}$ There are a number of risk factors for secondary failure, including patient-related factors such as long-standing disease, isolated small bowel disease, 


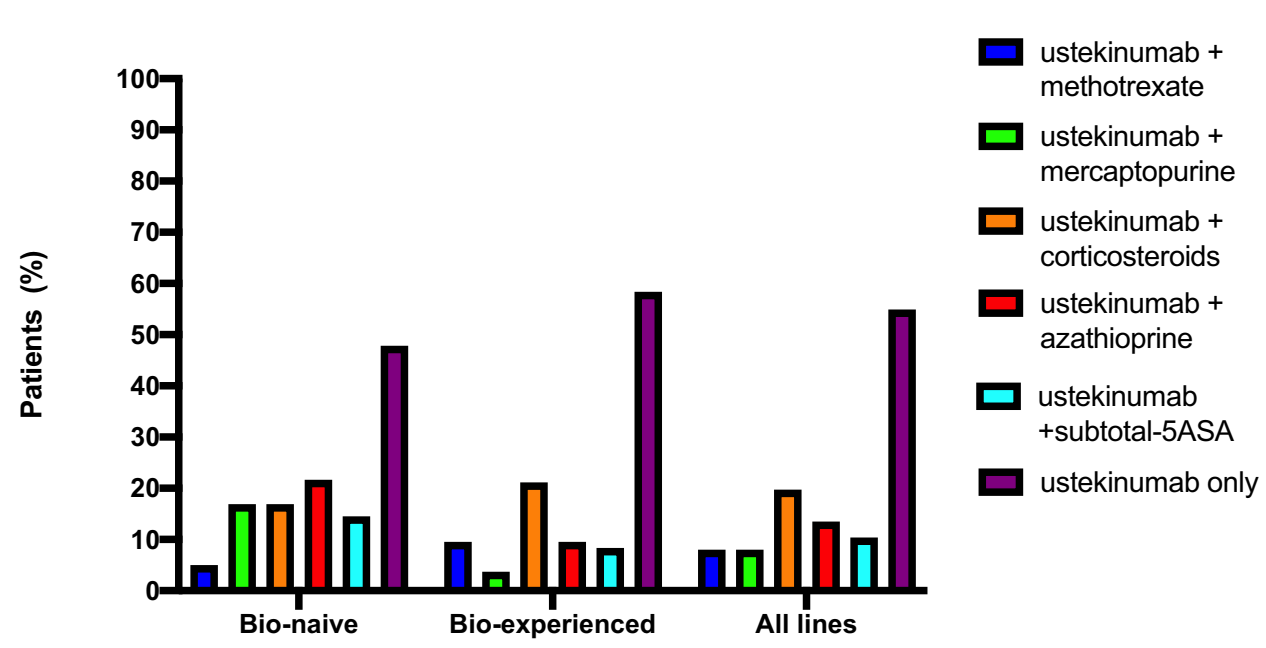

Figure 4 Percentage of patients receiving ustekinumab as a monotherapy or in combination by prior use of biologic therapy.

gastrointestinal involvement, severe intestinal inflammation and hypoalbuminemia. ${ }^{15}$ The influence of these factors on ustekinumab persistence has not yet been investigated. However, in this study, persistence was also high in biologically experienced patients with over $80 \%$ persistence at 12 months.

However, in patients with adequate anti-TNF drug concentrations and active disease, alternative pathways of inflammation (not TNF-driven) are likely; and therefore a drug with an alternative mechanism should be considered. ${ }^{15}$

In our study, males demonstrated a longer persistence at 12 months to females, which may be reflective of females ceasing biologics due to pregnancy considerations. Our study was unable to determine the underlying relationship between gender and persistence as there were limited demographic and clinical variables available. No significant differences in persistence by age were found for female patients. Anti-TNF drugs are Category C (drugs which have caused, or may be suspected of causing, harmful effects on the human fetus or neonate without causing malformations) whilst ustekinumab is pregnancy Category B1 (drugs which have been taken by only a limited number of pregnant women and women of childbearing age, without an increase in the frequency of malformation or other direct or indirect harmful effects on the human fetus having been observed). Anti-TNF drugs are prescribed to pregnant women. ${ }^{16}$ Whilst some research on the effects of biologic drugs in pregnancy has been done, this is an area that warrants further study.

There are a small number of comparable real-world studies of the persistence to biologics in $\mathrm{CD}$ from other jurisdictions. A systematic review of data from the United
States examined adherence, persistence, switching and dose escalation in patients treated for IBD with biologic therapies and determined variabilities among study design and methodology prevented meaningful comparisons. ${ }^{17}$ Discontinuation rates for patients with $\mathrm{CD}$ were found to range between $25 \%$ and $65 \%$ at 12 months. ${ }^{17}$ Persistence by line of therapy was not able to be meta-analyzed due to high variability between studies; however, persistence decreased with each subsequent line of therapy in one of the reviewed studies of anti-TNFs (mean persistence 21.5, 17.4 and 13.2 months respectively for first-, second- and third-line treatment). ${ }^{17}$ The main reasons for discontinuation were lack or loss of response, or adverse events.

Several jurisdictions have performed real-world studies of ustekinumab persistence in patients with $\mathrm{CD}$, including Finland ${ }^{18,19}$ and the $\mathrm{US}^{20}$ and reinforce the value of studying persistence to therapy. The US study included 214 patients, and linked patients' health resource utilization, medication changes and dosage changes via three national commercial databases. A similar 12-month persistence to our study was observed (84\%). ${ }^{20}$ Corticosteroid use also declined from $48 \%$ to $13 \%$ at the end of follow-up. ${ }^{18}$ There were fewer emergency department admissions. ${ }^{20}$ The most recent Finnish study reported high rates of persistence in 93 patients at 1 year $(83 \%) .{ }^{19}$ The study included 155 patients at 17 centers and patients showed a decrease from baseline to 1 year follow-up in the simple endoscopic score for Crohn's disease (SES-CD) from 10 to $3(\mathrm{P}=0.033), \mathrm{C}$-reactive protein from 7 to $5 \mathrm{mg} / \mathrm{L},(\mathrm{P}<$ $0.001)$ and fecal calprotectin from 776 to $305 \mu \mathrm{g} / \mathrm{g}$ (P < 0.001 , with the authors concluding significant reductions in disease activity and high long-term persistence. ${ }^{19}$ 
Our study has some limitations. Research using the $10 \%$ PBS sample is limited by the available sample size in the designated therapeutic area; no information is collated on disease severity or reason for discontinuation. In Australia, change to biologic therapy is possible for reasons other than treatment failure. The PBS sample includes community-based prescriptions and does not include medicine supplied under other schemes, or in hospitals or non-approved pharmacies. The available data is still relatively recent and more research is needed with more mature data to understand persistence in treatment in the real-world as well as the reasons for discontinuation. Despite these limitations, this remains an important study of Australian real-world data using a random 10\% sample of the population to describe the use of ustekinumab and confirm the findings of other real-world data globally.

\section{Conclusions}

This analysis reports the treatment persistence of ustekinumab in a real-world setting for both biologic-naïve and biologic experienced patients where there is a paucity of evidence on this topic. In Australia, strict PBS criteria must be satisfied before biologic therapies are prescribed. Our study observed a higher persistence for men vs women and a tendency towards higher persistence in the first line of therapy compared to later lines. There were no differences in treatment persistence between those administered ustekinumab as a monotherapy or combination therapy. Overall, persistence is in line with what has been reported in other studies around the world. Further studies comparing ustekinumab to other biologic drugs are planned in future once more long-term data are available for ustekinumab. These findings should provide confidence in the persistence of ustekinumab past 12 months, regardless of whether patients are administered concomitant CD therapy.

\section{Acknowledgments}

The authors thank Caroline Pile of WriteSource Medical Pty Ltd, Sydney, Australia, for providing medical writing support, which was funded by Janssen-Cilag Pty Ltd in accordance with Good Publication Practice (GPP3) guidelines (http://www.ismpp.org/gpp3).

\section{Funding}

This study was funded by Janssen-Cilag Pty Ltd, the manufacturer of ustekinumab.

\section{Disclosure}

AP, THC and THWH are employees of Janssen-Cilag Australia Pty Ltd. TK, MK and SC are employees of Prospection who were contracted by Janssen-Cilag to conduct this analysis. The authors report no other conflicts of interest in this work.

\section{References}

1. Australia, PricewaterhouseCoopers. Improving inflammatory bowel disease care across Australia. Commissioned by Crohns and Colitis Australia. 2013

2. Wilson J, Hair C, Knight R, et al. High incidence of inflammatory bowel disease in Australia: a prospective population-based Australian incidence study. Inflamm Bowel Dis. 2010;16(9):1550-1556. doi:10.1002/ibd.21209

3. Lamb CA, Kennedy NA, Raine T, et al. British Society of Gastroenterology consensus guidelines on the management of inflammatory bowel disease in adults. Gut. 2019;68(Suppl 3):s1-s106.

4. Alex GA, Bell S, Connor S, Moore G, Ward M, Vn Langenberg D. Australian guidelines for general practitioners and physicians inflammatory bowel disease 4th edition (updated 2018). Gastroenterol Soc Australia. 2018.

5. Janssen-Cliag. Stelara Ustekinumab Australian product information. 2020.

6. Torres J, Bonovas S, Doherty G, et al. ECCO guidelines on therapeutics in Crohn's disease: medical treatment. J Crohns Colitis. 2020;14(1):4-22. doi:10.1093/ecco-jcc/jjz180

7. Macaluso FS, Maida M, Ventimiglia M, Cottone M, Orlando A. Effectiveness and safety of Ustekinumab for the treatment of Crohn's disease in real-life experiences: a meta-analysis of observational studies. Expert Opin Biol Ther. 2020;20(2):193-203. doi:10.1080/14712598.2020.1707800

8. Kakkadasam Ramaswamy P, Moattar H, Sawyer E, Edwards J, Shukla D. P697 Efficacy and safety of ustekinumab in Crohn's disease: a real-world study from Australia. J Crohns Colitis. 2020;14 (Supplement_1):S564-S565. doi:10.1093/ecco-jcc/jjz203.825

9. Mellish L, Karanges EA, Litchfield MJ, et al. The Australian pharmaceutical benefits scheme data collection: a practical guide for researchers. BMC Res Notes. 2015;8(1):634. doi:10.1186/s13104-015-1616-8

10. Paige E, Kemp-Casey A, Korda R, Banks E. Using Australian pharmaceutical benefits scheme data for pharmacoepidemiological research: challenges and approaches. Public Health Res Pract. 2015;25(4). doi:10.17061/phrp2541546

11. Ko Y, Paramsothy S, Leong R. P361 The effect of immunomodulators and other factors on the persistence of biological agents for Crohn's disease and ulcerative colitis: data from the Australian population-based registry. J Crohns Colitis. 2020;14(Supplement_1): S342-S343. doi:10.1093/ecco-jcc/jjz203.490

12. Harris RJ, McDonnell M, Young D, et al. Early real-world effectiveness of ustekinumab for Crohn's disease. Frontline Gastroenterol. 2020;11(2):111-116. doi:10.1136/flgastro-2019-101237

13. Olivera P, Thiriet L, Luc A, Baumann C, Danese S, Peyrin-Biroulet L. Treatment persistence for infliximab versus adalimumab in Crohn's disease: a 14-year single-center experience. Inflamm Bowel Dis. 2017;23(6):976-985. doi:10.1097/MIB.0000000000001072

14. Dalal SR, Cohen RD. What to do when biologic agents are not working in inflammatory bowel disease patients. Gastroenterol Hepatol. 2015;11(10):657-665.

15. Fine S, Papamichael K, Cheifetz A. Etiology and management of lack or loss of response to anti-tumor necrosis factor therapy in patients with inflammatory bowel disease. Gastroenterol Hepatol. 2019;15(12):656-665. 
16. Flanagan E, Gibson PR, Rosella O, et al. P683 Infliximab, adalimumab and vedolizumab levels are not altered by pregnancy progression in IBD patients and neonatal vedolizumab levels are lower than in mothers: results from the PICCOLO study. J Crohns Colitis. 2020;14: S556-S557. doi:10.1093/ecco-jcc/jjz203.811

17. Khan S, Rupniewska E, Neighbors M, Singer D, Chiarappa J, Obando C. Real-world evidence on adherence, persistence, switching and dose escalation with biologics in adult inflammatory bowel disease in the United States: a systematic review. J Clin Pharm Ther. 2019;44(4):495-507. doi:10.1111/jcpt.12830

18. Eberl A, Hallinen T, Af Bjorkesten CG, et al. Ustekinumab for Crohn's disease: a nationwide real-life cohort study from Finland (FINUSTE). Scand J Gastroenterol. 2019;54(6):718-725. doi:10.1080/00365521. 2019.1624817
19. Af Björkesten CG, Ilus T, Hallinen T, et al. Objectively assessed disease activity and drug persistence during ustekinumab treatment in a nationwide real-world Crohn's disease cohort. Eur J Gastroenterol Hepatol. 2020;32(12):1507-1513. doi:10.1097/MEG.00000000000 01831

20. Obando C, Ding Z, Muser E, et al. Persistence, dose titration, and health care resource utilization among Crohn's disease patients treated with Ustekinumab: a real-world analysis in the United States. $A d v$ Ther. 2020;37(5):2127-2143. doi:10.1007/s12325-020-01276-3

\section{Publish your work in this journal}

Biologics: Targets and Therapy is an international, peer-reviewed journal focusing on the patho-physiological rationale for and clinical application of Biologic agents in the management of autoimmune diseases, cancers or other pathologies where a molecular target can be identified. This journal is indexed on PubMed Central, CAS, EMBase,
Scopus and the Elsevier Bibliographic databases. The manuscript management system is completely online and includes a very quick and fair peer-review system, which is all easy to use. Visit http://www.dovepress.com/testimonials.php to read real quotes from published authors. 